\title{
The Vaught Conjecture Do uncountable models count?
}

\author{
John T. Baldwin \\ Department of Mathematics, Statistics and Computer Science \\ University of Illinois at Chicago
}

July 12, 2006

\begin{abstract}
We give a model theoretic proof, replacing admissible set theory by the LopezEscobar theorem, of Makkai's theorem: Every counterexample to Vaught's conjecture has an uncountable model which realizes only countably many $L_{\omega_{1}, \omega}$-types. The following result is new. Theorem. If a first order theory is a counterexample to the Vaught conjecture then it has $2^{\aleph_{1}}$ models of cardinality $\aleph_{1}$.
\end{abstract}

In this paper we prove several properties of putative counterexamples to the Vaught conjecture. Specifically, these results concern the number of models the counterexample has in power $\aleph_{1}$. One of these results was proved 30 years ago using admissible model theory; we give a more straightforward argument. The following question guides our discussion. Is the Vaught Conjecture model theory?

Here are some possible ways in which this question would have a clear answer. Shelah, Buechler, Newelski have shown using rather difficult techniques from stability theory that the conjecture holds for first order theories that are 'simple' from the stability theoretic standpoint: $\omega$-stable or superstable with finite $U$-rank. If a counterexample were found for a first order theory of slightly greater complexity (e.g. a stable but not superstable first order theory), this would indicate the issue was a model theoretic one. If on the other hand, a uniform proof for sentences of $L_{\omega_{1}, \omega}$ were given using methods of descriptive set theory, then it would not be a model theoretic problem. The results below give partial answers to the following methodological questions.

What specific model theoretic as opposed to descriptive set theoretic techniques can attack the problem? Can one use more direct model theoretic arguments to obtain some result of admissible model theory?

I would argue the problem is model theoretic if its solution is different for $L_{\omega, \omega}$ and $L_{\omega_{1}, \omega}$. So we will investigate the differences between properties known about counterexamples to 
the Vaught Conjecture formulated in $L_{\omega, \omega}$ and $L_{\omega_{1}, \omega}$. Note that the Theorem of the abstract (for first order logic) is proved in ZFC; we ask whether it can be extended to $L_{\omega_{1}, \omega}$, perhaps with additional set theoretic hypotheses.

Much of model theory is concerned with models of arbitrary cardinality and with properties that in some way depend explicitly on cardinality. We pursue the theme, 'Do uncountable models count?' by noticing several results about the Vaught conjecture which revolve around the properties of uncountable models (and even the role of arbitrarily large models). Must a counterexample to $\mathrm{VC}$ in $L_{\omega_{1}, \omega}$ have a model of power $\aleph_{2}$ or even $\aleph_{1}$ ? Hjorth's contribution to this volume provides an answer to the last question- showing that if there is a counterexample to Vaught's conjecture then there is one with no model of cardinality $\aleph_{2}$.

In an attempt to clarify some techniques that are not widely known, we give more detail than is necessary in many cases. In Section 1, we provide some background on the nature of 'complete' sentences in $L_{\omega_{1}, \omega}$ and note that issues arise with both the upward and downward Löwenheim-Skolem theorem when generalizing to infinitary logic. We introduce the notion of a small uncountable model and note that stability theory for $L_{\omega_{1}, \omega}$ has been developed only for small models. And we relate Shelah's proof that this suffices for the study of categoricity; any sentence with few models in $\aleph_{1}$ has a small model. In Section 2, we make a brief excursion into Abstract Elementary Classes to illustrate quintessentially 'model theoretic' techniques. We use the results of Section 1 to provide a model theoretic proof of Makkai's theorem that any counterexample to Vaught's conjecture has an uncountable small model and in fact at least two models in power $\aleph_{1}$. In Section 3, we prove the (first order) theorem from the abstract and expound some old but not widely known results of Shelah about models of sentences of $L_{\omega_{1}, \omega}$ with cardinality at most $\aleph_{2}$; these results concern the ability to extend the result to $L_{\omega_{1}, \omega}$. We say a set $I$ is fully indiscernible in a model $M$ if every permutation of $I$ extends to an automorphism of $M$. Finally we use results of $\mathrm{Su}$ Gao to show that if a countable model $M$ admits an infinite fully indiscernible subset, then its Scott sentence has an uncountable model. This blocks some approaches to settling Vaught's conjecture.

\section{Complete Sentences and Small Uncountable Models}

Using both the upward and downward Löwenheim-Skolem theorem, it is easy to see that a first order theory that is categorical in some infinite cardinality is complete. The analog in the $L_{\omega_{1}, \omega}$-case requires some analysis. To begin with there are several possible meanings of complete depending on how much of $L_{\omega_{1}, \omega}$ is considered. In this first section, we stress this distinction and focus on the strongest such notion. We provide some non-trivial arguments for when models satisfy complete sentences and sketch some of the important consequences from a sentence being complete.

Let us formalize what constitutes a useful piece of $L_{\omega_{1}, \omega}$.

Definition 1.1 A fragment $\Delta$ of $L_{\omega_{1}, \omega}$ is a subset of $L_{\omega_{1}, \omega}$ closed under subformula, substi- 
tutions of terms, finitary logical operations and such that: whenever $\Theta \subset \Delta$ is countable and $\phi, \bigvee \Theta \in \Delta$ then $\bigvee\{\exists x \theta: \theta \in \Theta\}, \bigvee\{\phi \wedge \theta: \theta \in \Theta\}$, and $\bigvee(\{\phi\} \cup \Theta)$ are all in $\Delta$

Standard arguments show for every countable fragment $\Delta$ and every model $M$, there is a countable model $M^{\prime}$ that is $\Delta$-elementary submodel of $M$. Thus every satisfiable $L_{\omega_{1}, \omega^{-}}$ sentence has a countable model.

Definition 1.2 Let $\phi \in \Delta \subset L_{\omega_{1}, \omega}$ have a model.

1. $\phi$ is complete for $L_{\omega_{1}, \omega}$ (or just complete) if for every sentence $\psi$ of $L_{\omega_{1}, \omega}$, either $\phi \rightarrow \psi$ or $\phi \rightarrow \neg \psi$.

2. For any countable fragment $\Delta, \phi$ is complete for $\Delta$ if for every sentence $\psi \in \Delta$, either $\phi \rightarrow \psi$ or $\phi \rightarrow \neg \psi$.

This is an important distinction because in contrast to countable fragments the downward Löwenheim Skolem theorem is not true for arbitrary theories in $L_{\omega_{1}, \omega}$. In particular it is easy to find examples of uncountable structures which have no countable $L_{\omega_{1}, \omega}$-elementary submodel and so satisfy no complete sentence. Note that a sentence is complete if and only if it is a Scott sentence (a sentence of $L_{\omega_{1}, \omega}$ which completely describes a (countable) model).

A complete sentence of $L_{\omega_{1}, \omega}$ is $\aleph_{0}$-categorical, trivializing Vaught's conjecture. In Section 2 we will use $\Delta$-complete counterexamples to show that any counterexample to VC has both a model in $\aleph_{1}$ that satisfies a complete sentence and one that does not. In section 3 we will make crucial use of sentences that are complete to analyze the number of models in $\aleph_{1}$.

Definition 1.3 Let $\Delta$ be a fragment of $L_{\omega_{1}, \omega}$.

1. A model is $\Delta$-small if it realizes only countably many $\Delta$-types over the empty set.

2. A model is small if it realizes only countably many $L_{\omega_{1}, \omega}$-types over the empty set. That is, it is $\Delta$-small for $\Delta=L_{\omega_{1}, \omega}$.

Note that $M$ is small if and only $M$ is Karp-equivalent (i.e. $L_{\omega_{1}, \omega}$-equivalent) to a countable model. Thus any small model satisfies a complete sentence of $L_{\omega_{1}, \omega}$. The word small was suggested by the first order notion of a small theory: A first order theory is small if for every $n$, it has only countably many $n$-types over the empty set.

We first show that an $\aleph_{1}$-categorical sentence is implied by a complete sentence with a model of cardinality $\aleph_{1}$. We rely on the following result which combines results of LopezEscobar, Morley, and Keisler. The ingredients are in [8].

Theorem 1.4 Let $\tau$ be a similarity type which includes a binary relation symbol $<$. Suppose $\psi$ is a sentence of $L_{\omega_{1}, \omega}, M \models \psi$, and the order type of $(M,<)$ imbeds $\omega_{1}$. There is a model $N$ of $\psi$ with cardinality $\aleph_{1}$ such that the order type of $(N,<)$ imbeds $\mathbb{Q}$. 
Now we can prove:

Theorem 1.5 If the $L_{\omega_{1}, \omega}$-sentence $\psi$ has a model of cardinality $\aleph_{1}$ which is $\Delta$-small for every countable fragment $\Delta$ of $L_{\omega_{1}, \omega}$, then $\psi$ has a small model of cardinality $\aleph_{1}$.

Proof. If the $L_{\omega_{1}, \omega}-\tau$-sentence $\psi$ has a model of cardinality $\aleph_{1}$ which is $\Delta$-small for every countable $\tau$-fragment $\Delta$ of $L_{\omega_{1}, \omega}$, then $\psi$ has a $\tau$-small model of cardinality $\aleph_{1}$.

Add to $\tau$ a binary relation $<$, interpreted as a linear order of $M$ with order type $\omega_{1}$. Using that $M$ realizes only countably many types in any $\tau$-fragment, write $L_{\omega_{1}, \omega}(\tau)$ as a continuous increasing chain of fragments $L_{\alpha}$ such that each type in $L_{\alpha}$ realized in $M$ is a formula in $L_{\alpha+1}$.

Extend the similarity type to $\tau^{\prime}$ by adding new $2 n+1$-ary predicates $E_{n}(x, \mathbf{y}, \mathbf{z})$ and $n+1$-ary functions $f_{n}$.

Let $M$ satisfy $E_{n}(\alpha, \boldsymbol{a}, \mathbf{b})$ if and only if $\boldsymbol{a}$ and $\mathbf{b}$ realize the same $L_{\alpha}$-type.

Let $f_{n}$ map $M^{n+1}$ into the initial $\omega$ elements of the order, so that $E_{n}(\alpha, \boldsymbol{a}, \mathbf{b})$ implies $f_{n}(\alpha, \boldsymbol{a})=f_{n}(\alpha, \mathbf{b})$.

Notice the following facts.

1. $E_{n}(\beta, \mathbf{y}, \mathbf{z})$ refines $E_{n}(\alpha, \mathbf{y}, \mathbf{z})$ if $\beta>\alpha$;

2. $E_{n}(0, \boldsymbol{a}, \mathbf{b})$ implies $\boldsymbol{a}$ and $\mathbf{b}$ satisfy the same quantifier free $\tau$-formulas;

3. If $\beta>\alpha$ and $E_{n}(\beta, \boldsymbol{a}, \mathbf{b})$, then for every $c_{1}$ there exists $c_{2}$ such that $E_{n+1}\left(\alpha, c_{1} \boldsymbol{a}, c_{2} \mathbf{b}\right)$ and

4. $f_{n}$ witnesses that for any $a \in M$ each equivalence relation $E_{n}(a, \mathbf{y}, \mathbf{z})$ has only countably many classes.

All these assertions can be expressed by an $L_{\omega_{1}, \omega}\left(\tau^{\prime}\right)$ sentence $\phi$. Let $\Delta^{*}$ be the smallest $\tau^{\prime}$-fragment containing $\phi \wedge \psi$. Now by Lopez-Escobar (Theorem 1.4) there is a structure $N$ of cardinality $\aleph_{1}$ satisfying $\phi \wedge \psi \wedge \chi$ such that $<$ is not well-founded on $N$. Fix an infinite decreasing sequence $d_{0}>d_{1}>\ldots$ in $N$. For each $n$, define $E_{n}^{+}(\mathbf{x}, \mathbf{y})$ if for some $i$, $E_{n}\left(d_{i}, \mathbf{x}, \mathbf{y}\right)$. Now using 1), 2) and 3) prove by induction on the quantifier rank of $\phi$ for every $L_{\omega_{1}, \omega}(\tau)$-formula $\phi$ that $N \models E_{n}^{+}(\boldsymbol{a}, \mathbf{b})$ implies $N \models \phi(\boldsymbol{a})$ if and only if $N \models \phi(\mathbf{b})$.

For each $n, E_{n}\left(d_{0}, \mathbf{x}, \mathbf{y}\right)$ refines $E_{n}^{+}(\mathbf{x}, \mathbf{y})$ and by 4$) E_{n}\left(d_{0}, \mathbf{x}, \mathbf{y}\right)$ has only countably many classes; so $N$ is small.

Shelah 'reduces' Morley's categoricity theorem for $L_{\omega_{1}, \omega}$ to complete sentences. This reduction involves a crucial model theoretic technique: Prove a theorem for arbitrary vocabularies $\tau$. In fact, as exemplified in the next theorem, this reduction applies to more general questions concerning the number of models in $\aleph_{1}$ if the sentence has few models in $\aleph_{1}$. 
Theorem 1.6 Let $\psi$ be a complete sentence in $L_{\omega_{1}, \omega}$ in a countable vocabulary $\tau$. Then there is a countable vocabulary $\tau^{\prime}$ extending $\tau$ and a first order $\tau^{\prime}$-theory $T$ such that reduct is a 1-1 map from the atomic models of $T$ onto the models of $\psi$.

If $\psi$ is not complete, the reduction is only to 'finite diagrams' [15]. This is a very important distinction, the arguments given in Section 3 depend heavily on working in an atomic class. This 'reduction' is not direct. In order to deduce categoricity for an arbitrary $L_{\omega_{1}, \omega}$-sentence, stronger results than transfer of categoricity must be proved for complete $L_{\omega_{1}, \omega}$ sentences $([18]$ expounded in $[3,1])$.

There are two different arguments to obtain this reduction. If the sentence $\psi$ has arbitrarily large models the result is is a fairly straightforward argument with Ehrenfeucht-Mostowski models.

Theorem 1.7 Let $\psi$ be an $L_{\omega_{1}, \omega}(\tau)$-sentence which has arbitrarily large models. If $\psi$ is categorical in some cardinal $\kappa, \psi$ is implied by a consistent complete sentence $\psi^{\prime}$, which has a model of cardinality $\kappa$.

Without the arbitrarily large models assumption, the argument is considerably more difficult; it relies relies on both Theorem 1.5 and the following theorem of Keisler [8]. Model theoretically, these version are somewhat stronger; they need only few rather one model in cardinality $\aleph_{1}$.

Theorem 1.8 For any $L_{\omega_{1}, \omega}$-sentence $\psi$ and any fragment $\Delta$ containing $\psi$, if $\psi$ has fewer than $2^{\aleph_{1}}$ models of cardinality $\aleph_{1}$ then for any $M \models \psi$ of cardinality $\aleph_{1}, M$ realizes only countably many $\Delta$-types over the empty set

Theorem 1.9 Let $\psi$ be an $L_{\omega_{1}, \omega}(\tau)$-sentence. If $\psi$ has fewer than $2^{\aleph_{1}}$ models of cardinality $\aleph_{1}, \psi$ is implied by a consistent complete sentence $\psi^{\prime}$, which has a model of cardinality $\aleph_{1}$.

Proof. By Theorem 1.8, there is a model of power $\aleph_{1}$ which is $\Delta$-small for every countable fragment $\Delta$. But then by Theorem 1.5, there is an uncountable small model $N$ of $\psi$ and the Scott sentence of $N$ is as required.

\section{Two Models in $\aleph_{1}$}

In this section we reprove (in one case much more simply) old theorems showing that a counterexample to Vaught's conjecture must have two models in power $\aleph_{1}$. But we first take a brief excursion through Abstract Elementary Classes to see what I take as the essence of 'model theoretic' methods - arguments involving the direct constructions of models- apply in this context. 
Vaught's conjecture concerns the set of countable models of a 'theory'. An Abstract Elementary Class (AEC) is one of the most abstract formulations of 'theory' [21, 20, 5, 1]. A class of $L$-structures and a notion of 'strong submodel' $\prec,(\boldsymbol{K}, \prec)$, is said to be an abstract elementary class if both $\boldsymbol{K}$ and the binary relation $\prec$ are closed under isomorphism and satisfy a collection of conditions generalizing those of Jónnson for constructing homogeneous universal models. In particular, the class must be closed under $\prec$-increasing chains. The class is presented as a collection of models and a further crucial requirement is the existence of a Löwenheim number for the class.

So an extreme form of 'Vaught's conjecture is model theory' would be to prove it for any AEC. But this fails. The set $\boldsymbol{K}=\left\{\alpha: \alpha \leq \aleph_{1}\right\}$ with $\prec$ as initial segment is an AEC with $\aleph_{1}$ countable models. But the counterexample has no large models. (The Löwenheim number requirement forbids using all ordinals as the example.) The upward LowenheimSkolem theorem is true for $L_{\omega, \omega}$ but not $L_{\omega_{1}, \omega}$. So this excursion into the abstract leads us to some more precise questions.

In the absence of the upward Löwenheim-Skolem theorem, how can one construct models of larger cardinality? For the moment we continue in the context of AEC.



We begin by mentioning some fairly easy principles. Much more technical arguments are needed to obtain the hypotheses of these lemmas. An extendible model is more often referred to as one which non-maximal. Obviously,

Lemma 2.2 In any $A E C$, if every model of size $\lambda$ is $\prec$-extendible, there is a model of size $\lambda^{+}$.

Since AEC's are closed under unions of chains, in any AEC, if there is a strictly increasing $\prec$-sequence $M_{\alpha}, \alpha<\lambda^{+}$of models of size $\lambda$, there is a model of size $\lambda^{+}$. Even more,

Lemma 2.3 If the AEC $\boldsymbol{K}$ is $\lambda$-categorical and the model of size $\lambda$ is extendible then there is a model of cardinality $\lambda^{+}$.

Now we specialize to studying $L_{\omega_{1}, \omega}$-counterexamples to Vaught's conjecture. The existence of Scott sentences guarantee that if there is a countable $\prec$-extendible model it has an uncountable $L_{\omega_{1}, \omega}$-elementary extension. We sketch the analysis of Harnik and Makkai [6] to show every counterexample to VC has an uncountable 'large' (not small) model. For this they introduce another technical meaning for large; now describing a sentence rather than a model.

Definition 2.4 A sentence $\sigma$ of $L_{\omega_{1}, \omega}$ is large if it has uncountably many countable models. A large sentence $\sigma$ is minimal if for every sentence $\phi$ either $\sigma \wedge \phi$ or $\sigma \wedge \neg \phi$ is not large. 
By a tree argument [6] show:

Lemma 2.5 (Harnik-Makkai) For every counterexample $\sigma$ to Vaught's conjecture, there is a minimal counterexample $\phi$ such that $\phi \models \sigma$.

Our first goal is to show any counterexample to Vaught's conjecture has an uncountable model which is not small. Fix a minimal counterexample $\sigma$ to Vaught's conjecture. For any countable fragment $\Delta$ containing $\sigma$, define

$$
T_{\Delta}=\{\sigma \wedge \phi: \phi \in \Delta \text { and } \sigma \wedge \phi \text { is large }\}
$$

Note that $T_{\Delta}$ is consistent and complete for $\Delta$. Keisler [8] with no use of admissible model theory shows that the 'prime' part of Vaught's fundamental paper on countable models of complete first order theories [22] goes through for scattered $\sigma$. This translation is fairly straightforward without any appeal to admissible model theory. In particular,

Fact 2.6 A theory $T$ that is complete for a countable fragment of $L_{\omega_{1}, \omega}$ and has only countably many types over the empty set has a prime model.

Since $\sigma$ is scattered, each $T_{\Delta}$ has a prime model (for $\Delta$ ).

Lemma 2.7 If $\sigma$ is a counterexample to the Vaught Conjecture and $\Delta$ is the smallest fragment containing $\sigma$, there is a strictly increasing $\prec_{\Delta}$-sequence $M_{\alpha}, \alpha<\aleph_{1}$ of countable models.

Proof. Fix a minimal counterexample $\sigma$ to Vaught's conjecture and let $\Delta_{0}$ be a countable fragment containing $\sigma\left(\{\sigma\}=T_{0}\right)$. Define by induction $\left\langle\Delta_{\alpha}, T_{\alpha}, M_{\alpha}\right\rangle$ such that

1. If $\beta<\alpha$, the Scott sentence $\psi_{\beta}$ of $M_{\beta}$ is in $\Delta_{\alpha}$.

2. $T_{\alpha}=T_{\Delta_{\alpha}}$

3. $M_{\alpha}$ is the $\Delta_{\alpha}$ prime model of $T_{\alpha}$.

For this, let $\Delta_{\alpha}$ be the minimal fragment containing $\bigcup_{\beta<\alpha} \Delta_{\beta}$ and the Scott sentence of each $M_{\beta}$ for $\beta<\alpha$. The $M_{\alpha}$ are as required. The chain is strictly increasing since $M_{\alpha}=\neg \psi_{\beta}$ if $\beta<\alpha$. And each $M_{\alpha} \prec_{\Delta_{0}} M_{\beta}$ for $\alpha<\beta$ since the $\Delta_{i}$ and $T_{i}$ are increasing. That is, $M_{\alpha}$ is the prime model of $T_{\alpha}$ and $M_{\beta} \models T_{\alpha}$.

$\square 2.7$

Theorem 2.8 (Harnik-Makkai) If $\sigma \in L_{\omega_{1}, \omega}$ is a counterexample to $V C$ then it has a model $N$ of cardinality $\aleph_{1}$ which is not small. 
Proof. We continue the argument from Lemma 2.7. Now if $M=\bigcup_{\alpha} M_{\alpha}, M$ does not satisfy any complete sentence of $L_{\omega_{1}, \omega}$, as any sentence $\theta$ true on $M$ is true on a cub of $M_{\alpha}$; so it has more than one countable model and cannot be complete. But since every small model is satisfied by a complete sentence, $M$ is not small.

Our goal now is to show that any counterexample to Vaught's conjecture has small uncountable models. This was first obtained by Makkai, using (in contrast to Keisler's study of prime models) notions of saturated models in admissible set theory and some reasonably elaborate machinery devised by Ressayre [14] (basic to admissible model theory but much more than we will use here). Now we apply Theorem1.5 to provide a proof which trades the mechanism of admissible sets for a model theoretic coding to analyze models of cardinality $\aleph_{1}$.

Note that by the downward Löwenheim-Skolem theorem every model of a complete sentence of $L_{\omega_{1}, \omega}$ is small. So every $L_{\omega_{1}, \omega}$-complete sentence is scattered in the following sense.

Definition 2.9 1. $S_{n}(\sigma, \Delta)$ denotes the collection of $n$-types in $\Delta$ that are realized in models of $\sigma$.

2. A sentence $\sigma$ of $L_{\omega_{1}, \omega}$ is scattered if for every countable fragment $\Delta$ of $L_{\omega_{1}, \omega}, S_{n}(\sigma, \Delta)$ is countable for each $n$.

If $\sigma$ is scattered and $\sigma^{\prime} \rightarrow \sigma$, then $\sigma^{\prime}$ is scattered. In his landmark proof that a counterexample to Vaught's conjecture has at most $\aleph_{1}$ models of cardinality $\aleph_{0}$, Morley [13] established, by essentially descriptive set theoretic arguments:

Theorem 2.10 (Morley) If $\sigma$ is a counterexample to VC, $\sigma$ is scattered.

Note that the hypothesis of Theorem 1.5 is satisfied by any scattered $L_{\omega_{1}, \omega}$-sentence that has an uncountable model.

We conclude the result proved by Makkai[11] using admissible model theory.

Theorem 2.11 (Makkai) If $\sigma \in L_{\omega_{1}, \omega}$ is a counterexample to VC then it has an uncountable model $N$ which is small.

Proof. By Lemma 2.10, $\psi$ is scattered. By Theorem 2.8, it has a model of power $\aleph_{1}$ and then by Lemma 1.5, it has a small uncountable model.

We have shown:

Corollary 2.12 There is no $\aleph_{1}$-categorical counterexample to Vaught's conjecture. 
We detour to briefly to discuss an alternative very natural approach to constructing small models of cardinality $\aleph_{1}$. The next lemma also emphasizes why we spoke of $\prec$-extendible rather than just extendible models; the precise notion of 'elementary equivalence' is very important. Note that with the choice of $\Delta$ below, $\prec_{\Delta}$ is the same as saying $L_{\infty, \omega}$-elementary.

Lemma 2.13 A sentence $\sigma$ of $L_{\omega_{1}, \omega}$ has an uncountable small model iff it has a pair of countable models such that $M_{0}$ is a proper substructure of $M_{1}, M_{0}$ and $M_{1}$ are isomorphic and $M_{0} \prec_{\Delta} M_{1}$, where $\Delta$ is the smallest fragment containing the Scott sentence of $M_{0}$.

Proof. If $N$ is an uncountable small model of $\sigma$, let $\psi$ be the Scott sentence of $N$ and $L$ the fragment generated by $\psi$. Then take $M_{0}$ an $L$-elementary submodel of $N$ and $M_{1}$ an $L$-elementary submodel of $N$ which properly extends $M_{0}$. Conversely, construct an chain $\left\langle M_{i}: i<\aleph_{1}\right\rangle$ where $\left(M_{i}, M_{i+1}\right)$ is isomorphic to $\left(M_{0}, M_{1}\right)$. This construction goes through limits by taking unions since for countable $\delta$, all $M_{\delta}$ are isomorphic. Then every type realized in $M_{\omega_{1}}$ is realized in $M_{0}$ so it is a small uncountable model of $\psi$. $\square_{2.13}$

Continuing our methodological queries, is there any direct way (using only countable

models) to deduce the existence of such a pair of countable models directly from the failure of Vaught's conjecture?

During the conference Sacks sketched a positive reply to this question by a nice argument using admissible sets and Barwise compactness which gave the result via a construction on countable models. In essence Makkai's original argument [11] also provides a positive answer using the technology of admissible set theory.

\section{The number of models in $\aleph_{1}$}

We have shown that any counterexample to Vaught's conjecture has at least two models of cardinality $\aleph_{1}$. Why stop there? The following result seems to be new.

Theorem 3.1 If a first order theory is a counterexample to the Vaught conjecture then it has $2^{\aleph_{1}}$ models of cardinality $\aleph_{1}$.

But it is easy from two well-known but difficult theorems:

Theorem 3.2 (Shelah) If a first order $T$ is not $\omega$-stable $T$ has $2^{\aleph_{1}}$ models of cardinality $\aleph_{1}$.

This argument uses many descriptive set theoretic techniques. See Shelah's book [17] or Baldwin's paper [2].

Theorem 3.3 (Shelah) An $\omega$-stable first order theory satisfies Vaught's conjecture. 
Proof of 3.1: If $T$ has less than $2^{\aleph_{1}}$ models of cardinality $\aleph_{1}$ then by Theorem 3.2 , it is $\omega$-stable and then by Theorem 3.3, it satisfies Vaught's conjecture. $\quad \square_{3.1}$

We now discuss the possibility of assuming the weak continuum hypothesis $\left(2^{\aleph_{0}}<2^{\aleph_{1}}\right)$, to extend the previous theorem to $L_{\omega_{1}, \omega}$. This provides an excuse for describing the role of the weak continuum hypothesis in some nice constructions of Shelah and Keisler concerning the spectrum of sentences of $L_{\omega_{1}, \omega}$. We say that a complete sentence $\sigma$ in $L_{\omega_{1}, \omega}$ is $\omega$-stable if only countably many $L_{\omega_{1}, \omega}$-types over any countable $M \models \sigma$ are realized in some model of $\sigma$. This is a strictly and crucially weaker assumption than if we replace the countable model $M$ by a countable set $A$. Shelah observed that under the weak continuum hypothesis, Theorem 1.8, which asserted that few models in $\aleph_{1}$ yields few types over the empty set, implies:

Fact $3.4\left(2^{\aleph_{0}}<2^{\aleph_{1}}\right)$ If a sentence $\psi \in L_{\omega_{1}, \omega}$ is not $\omega$-stable it has $2^{\aleph_{1}}$ models of cardinality $\aleph_{1}$.

As noted above, for first order logic, few models in $\aleph_{1}$ implies $\omega$-stable. And this result even holds (in ZFC) for sentences in $L_{\omega_{1}, \omega}$, which have arbitrarily large models. The arbitrarily large models give us access to Ehrenfeucht-Mostowski models. But for an arbitrary sentence in $L_{\omega_{1}, \omega}$, to show few models in $\aleph_{1}$ implies $\omega$-stable, requires weak CH. Shelah [16] first provided a counterexamples in $L_{\omega, \omega}(Q)$ using Baumgartner's order. But examples can be found in $L_{\omega_{1}, \omega}[21,1]$.

This leads us to some natural generalization of Theorem 3.3. The notion of an excellent class $[18,19,3,23]$ plays a crucial role in the model theory of infinitary logic.

Question 3.5 Does Vaught's conjecture hold for $\omega$-stable sentences in $L_{\omega_{1}, \omega}$ ? For excellent classes?

These questions pose two difficulties. As Rami Grossberg pointed out the questions are not really well-formed. The work in $[18,19]$ on $\omega$-stable and excellent classes is restricted to atomic classes - the translation of complete sentences of $L_{\omega_{1}, \omega}$. All such classes are $\aleph_{0^{-}}$ categorical. So the first step is to adapt the stability theory machinery for the translations of arbitrary sentences in $L_{\omega_{1}, \omega}$. These are finite diagrams in the sense of [15]. But the machinery of that paper is primarily directed at the study of uncountable models and makes the additional assumption that there is a homogeneous model. Once an appropriate framework is found that circumvents these difficulties, the real task begins. The proof that an $\omega$-stable first order theory has either $\aleph_{0}$ or $2^{\aleph_{0}}$ countable models has two parts. On the one hand various conditions are shown to imply the existence of $2^{\aleph_{0}}$ countable models; on the other the conjunction of the negations of these properties are shown to allow such control over the structure of models that the theory has only countably many models. This second part might be easier with the greater expressive power of $L_{\omega_{1}, \omega}$. But the loss of compactness may greatly complicate the first. 
Many of the difficulties in studying $L_{\omega_{1}, \omega}$ stem from the difficulty of proving the amalgamation property. Recall that a sentence $\sigma$ in a fragment $\Delta$ of $L_{\omega_{1}, \omega}$ satisfies the amalgamation property if $M_{0} \prec_{\Delta} M_{1}, M_{2}$ implies $M_{1}$ and $M_{2}$ have a common $\Delta$-elementary extension.

Theorem 3.6 (Shelah) $\left(2^{\aleph_{0}}<2^{\aleph_{1}}\right)$ If a sentence $\sigma$ in $L_{\omega_{1}, \omega}$ has fewer than $2^{\aleph_{1}}$ models of cardinality $\aleph_{1}$ then the countable models of $\sigma$ have the amalgamation property.

The argument for this can be found in $[21,5,1]$. The weak $\mathrm{CH}$ is used to apply the Devlin-Shelah diamond; this use is necessary and counterexamples are in the same place. Consider the following theorem of Shelah.

Theorem 3.7 An $\aleph_{1}$-categorical sentence $\psi$ in $L_{\omega_{1}, \omega}$ has a model of power $\aleph_{2}$.

This result actually was first proved in more generality for $L_{\omega_{1}, \omega}(Q)$ (adding the quantifier, 'there exists uncountably many'), but for Vaught conjecture considerations we restrict to $L_{\omega_{1}, \omega}$. The original proof [16] used diamond and developed a considerable amount of stability theory for $L_{\omega_{1}, \omega}$. In ([21], see also [1]) a beautiful proof of Theorem 3.7 is given in ZFC. The crux is to use another application of Lopez-Escobar to construct a proper pair of cardinality $\aleph_{1}$. Then as in Lemma 2.3, categoricity shows every model of power $\aleph_{1}$ is extendible and so yields a model in $\aleph_{2}$. The argument below weakens categoricity to few models in $\aleph_{1}$. The condition that there is some proper pair in $\aleph_{1}$ is strengthened to showing there is no maximal model of power $\aleph_{1}$ and then the model of power $\aleph_{2}$ follows as in Lemma 2.2.

By the reductions of Section 2.2, we may work with an atomic class: the class of atomic models of a complete first order theory. In the next theorem, which appears to be newly remarked (although of course implicit in [18] if not [16]), we weaken the hypothesis of $\aleph_{1^{-}}$ categoricity in Theorem 3.7 to $\omega$-stability; we are still working in ZFC.

As in $[16,18]$ and expounded in $[1,10]$, we develop the notion of an $\omega$-stable atomic class. (Warning, many words (type, $\omega$-stable, independent etc.) have subtly different meanings in this context. So new arguments are needed for what at first appear to be old results.) Most crucially, all amalgamation questions are slippery. A notion of independence, $M \underset{N}{\downarrow} P$, is defined (based on splitting), which has many of the properties of the first order notion of 'nonforking'. One is able to show that countable models in $\boldsymbol{K}$ admit a form of free amalgamation. See the chapter on independence in $\omega$-stable atomic classes of [1] for a recent detailed exposition of the next few theorems.

Definition 3.8 $A$ and $B$ are freely amalgamated over $N$ in $M$, if $A B \subset M \in \boldsymbol{K}$ and $A \downarrow B$. $N$

Fact 3.9 If $M_{0} \prec M_{1}, M_{2}$ then there exists $M_{1}^{\prime} \approx M_{1}$ and $M_{3}$ with $M_{1}^{\prime}$ and $M_{2}$ freely amalgamated over $M_{0}$ in $M_{3}$. 
Theorem 3.10 If the atomic class is $\omega$-stable and has a model of power $\aleph_{1}$ then it has a model of power $\aleph_{2}$.

Proof. As in Lemma 2.2, it suffices to show every model $N$ in $\boldsymbol{K}$ of cardinality $\aleph_{1}$ has a proper elementary extension $M$ in $\boldsymbol{K}$. Write $N$ as a continuous increasing chain $\left\langle N_{i}: i<\aleph_{1}\right\rangle$. By Theorem 3.2, $\boldsymbol{K}$ is $\omega$-stable. Now define an increasing sequence $\left\langle M_{i}: i\left\langle\aleph_{1}\right\rangle\right.$ such that $N_{i} \prec M_{i}, M_{i}$ is freely amalgamated with $N_{i+1}$ over $N_{i}$ in $M_{i+1}$. Since independent sets intersect only where they have to, $M_{0}$ properly extends $N_{0}$. The union of the $M_{i}$ is the required proper extension of $N$. The construction is routine taking unions at limits. The successor stage is also easy from the following claim (which can be proved for $\omega$-stable atomic classes), replacing $0,1,2$ by $\alpha, \alpha+1, \alpha+2$ but keeping $N$ fixed.

Claim 3.11 Let $N_{0} \prec N_{1} \prec N_{2} \prec N$. Given $M_{0} \underset{N_{0}}{\downarrow} N_{2}$, with $M_{0}$ and $N_{1}$ freely amalgamated over $N_{0}$ in $M_{2}^{\prime}$, we can choose $M_{2}$ and $M_{3}^{\prime}$ so that $N_{2}, M_{1} \prec M_{2}$ and $M_{2}$ and $N_{2}$ are freely amalgamated over $N_{1}$ in $M_{3}^{\prime}$.

The hypothesis in Theorem 3.10 that there be a model with cardinality $\aleph_{1}$ is essential. As defined here, the Marcus example [12] is $\omega$-stable and has exactly one model.

Now we can strengthen Theorem 3.7 replacing categoricity in $\aleph_{1}$ by few models in $\aleph_{1}$ at the cost of assuming $2^{\aleph_{0}}<2^{\aleph_{1}}$. The following corollary is immediate since with this set-theoretic hypothesis, few models in $\aleph_{1}$ implies $\omega$-stability (Lemma 3.2).

Corollary 3.12 (Shelah) $\left[2^{\aleph_{0}}<2^{\aleph_{1}}\right]$ If the atomic class $\boldsymbol{K}$ has at least one but fewer than $2^{\aleph_{1}}$ models of cardinality $\aleph_{1}$ then it has a model of power $\aleph_{2}$.

Recall Hjorth [7] proved:

Theorem 3.13 (Hjorth) If there is a counterexample to Vaught's conjecture, there is one with no model of size $\aleph_{2}$.

Note that by Lemma 3.12, under the weak continuum hypothesis we deduce that Hjorth's example has $2^{\aleph_{1}}$ models of cardinality $\aleph_{1}$. The number of models in $\aleph_{1}$ does not appear to be controlled by Hjorth's construction. This leads to a number of specific problems.

1. Show Hjorth's example has $2^{\aleph_{1}}$ models in $\aleph_{1}$ in ZFC.

2. Can one just prove directly that any counterexample to Vaught's conjecture has $2^{\aleph_{1}}$ models of cardinality $\aleph_{1}$ ? 
A natural strategy for the second question is to return to the initial Harnik-Makkai argument, Lemma 2.8, and code stationary sets into the construction of the tree. But this requires some notion of how different 'tops' are put on the limits of countable chains and there is nothing of this sort evident (to me) in the proof. And such an argument might not avoid the set theory since Devlin-Shelah diamond is used in many such arguments. Moreover, the proof of the first order case involves a deep analysis of the models; it would be very striking to avoid this.

If one showed any counterexample to Vaught's conjecture has $2^{\aleph_{1}}$ models of cardinality $\aleph_{1}$, then a strategy to solve Vaught's conjecture would be to show that if there is a counterexample to Vaught's conjecture, then there is one with $\aleph_{1}$ models of cardinality $\aleph_{1}$. And there is a marvelously simple recipe for such an example. Marcus constructed [12] a first order theory $T$ with an atomic model that has no elementary submodel but contains a definable subset $P$ comprising an infinite set of indiscernibles. Now impose a structure (from a disjoint language) on $P$ and require that this structure is a model of the counterexample to Vaught's conjecture. The disjoint union of this model with a pure set seems to have $\aleph_{1}$ models of power $\aleph_{1}$. But this holds only if 'indiscernible' is read as 'fully indiscernible' in the following sense.

Definition 3.14 A set I is fully indiscernible in a model $M$ if every permutation of $I$ extends to an automorphism of $M$.

We defeat the pipe dream above with the following corollary to work of Su Gao [4]. The crux is a characterization of ' $M$ is extendible (for $L_{\omega_{1}, \omega}$ ) in terms of the automorphism group of $M$. Kueker [9] showed that $|\operatorname{aut}(M)|=2^{\omega}$ if $M$ is an extendible countable model but the converse fails. In line with our previous methodological standpoint we will sketch some of Gao's argument for a characterization rather than just quoting the final result to distinguish model theoretic and descriptive set theoretic techniques used in the argument. The various descriptive set theoretic assertions in the next couple of paragraphs are proved in [4].

Proposition 3.15 If a countable model $M$ admits an infinite subset of full indiscernibles then $M$ is extendible (for $L_{\omega_{1}, \omega}$ ).

Proof. We first note that $M$ is extendible if and only if aut $(M)$ is closed in $\omega^{\omega}$. To see this, rephrase 'extendible' as 'there exists an $(\infty, \omega)$ map $j$ from $M$ properly into itself. (This relies on $\prec$ being $(\infty, \omega)$-submodel and the countability of $M$.) Clearly, if aut $(M)$ is not closed in $\omega^{\omega}$, there is a sequence $f_{n}$ of automorphisms of $M$ whose pointwise limit is an $(\infty, \omega)$ map (so clearly an injection) but not an automorphism, i.e. not onto. In the other direction, given such a map $j$; enumerate $M$ as $\left\langle a_{i}: i<\omega\right\rangle$. Then for each $n, a_{1}, \ldots a_{n}$ and $j a_{1}, \ldots j a_{n}$ are $(\infty, \omega)$-equivalent so $j\left\lceil a_{1}, \ldots a_{n}\right.$ extends to an automorphism $f_{n}$ of $M$. The sequence $f_{n}$ verifies that $\operatorname{aut}(M)$ is not closed.

So far the argument is model theoretic although the conclusion about the topology on Baire Space is crossing the line. But even more, Gao restates the condition as follows: 
Fact 3.16 If $G$ is a closed subgroup of $S_{\infty}$, then $G$ admits a left-invariant complete metric if and only if $G$ is closed in the Baire space $\omega^{\omega}$.

And to complete the proof, we use these abstract conditions on the automorphism group. Suppose $I$ is fully indiscernible in a model $M$. Then aut $(M)$ projects (by restriction) onto the group of permutations of $I, S_{\infty}$. Thus, if $M$ is not extendible, $G=\operatorname{aut}(M)$ is closed in $\omega^{\omega}$ and so admits a left-invariant complete metric. But then, as Gao further shows, the projection of $G$ onto $S_{\infty}$ would induce a left-invariant complete metric on $S_{\infty}$. There is no such metric and we finish.

$\square_{3.15}$

This conference exhibited a striking interaction among logicians of various stripes. This paper is one example; I raised the question of whether a counterexample to Vaught's conjecture necessarily had a model of cardinality $\aleph_{2}$ early in the conference; Sacks elaborated on the question in his second presentation; Hjorth heard the problem in Sack's lecture and had the tools to solve it. And in writing up my contribution, I saw that Hjorth's solution suggests some new strategies for attacking Vaught's conjecture itself. But these strategies are restrained by Gao's results which are shown by a real interweaving of model theoretic and descriptive set theoretic techniques.

\section{References}

[1] John T. Baldwin. Categoricity. www.math.uic.edu/ jbaldwin.

[2] J.T. Baldwin. Diverse classes. Journal of Symbolic Logic, 54:875-893, 1989.

[3] J.T. Baldwin. Notes on quasiminimality and excellence. Bulletin of Symbolic Logic, 10:334-367, 2004.

[4] Su Gao. On automorphism groups of countable structures. The Journal of Symbolic Logic, 63:891-896, 1996.

[5] Rami Grossberg. Classification theory for non-elementary classes. In Yi Zhang, editor, Logic and Algebra, pages 165-204. AMS, 2002. Contemporary Mathematics 302.

[6] V. Harnik and M. Makkai. A tree argument in infinitary model theory. Proceeedings of the American Mathematical Society, 67:309-314, 1977.

[7] Greg Hjorth. A note on counterexamples to Vaught's conjecture. preprint; this volume, 200x.

[8] H.J Keisler. Model theory for Infinitary Logic. North-Holland, 1971. 
[9] D. W. Kueker. Back and forth arguments and infinitary model theory. In D. W. Kueker, editor, Infinitary Logic: In Memoriam Carol Karp, pages 17-72. Springer-Verlag, 1970. LNM 492.

[10] Olivier Lessmann. An introduction to excellent classes. In Yi Zhang, editor, Logic and its Applications, Contemporary Mathematics, pages 231-261. American Mathematical Society, 2005.

[11] M. Makkai. An 'admissible generalization of a theorem on countable $\sigma_{1}^{1}$ sets of reals with applications. Annals of Mathematical Logic, 11:1-30, 1977.

[12] L. Marcus. A minimal prime model with an infinite set of indiscernibles. Israel Journal of Mathematics, 11:180-183, 1972.

[13] M. Morley. The number of countable models. The Journal of Symbolic Logic, 35:14-18, 1970 .

[14] J.-P. Ressayre. Models with compactness properties relative to an admissible language. Annals of Mathematical Logic, 11:31-55, 1977.

[15] S. Shelah. Finite diagrams stable in power. Annals of Mathematical Logic, 2:69-118, 1970 .

[16] S. Shelah. Categoricity in $\aleph_{1}$ of sentences in $L_{\omega_{1}, \omega}(Q)$. Israel Journal of Mathematics, 20:127-148, 1975. paper 48.

[17] S. Shelah. Classification Theory and the Number of Nonisomorphic Models. NorthHolland, 1978.

[18] S. Shelah. Classification theory for nonelementary classes. I. the number of uncountable models of $\psi \in L_{\omega_{1} \omega}$ part A. Israel Journal of Mathematics, 46:3:212-240, 1983. paper 87 a.

[19] S. Shelah. Classification theory for nonelementary classes. I. the number of uncountable models of $\psi \in L_{\omega_{1} \omega}$ part B. Israel Journal of Mathematics, 46;3:241-271, 1983. paper $87 \mathrm{~b}$.

[20] S. Shelah. Categoricity for abstract classes with amalgamation. Annals of Pure and Applied Logic, 98:261-294, 1999. paper 394. Consult Shelah for post-publication revisions.

[21] Saharon Shelah. Classification of nonelementary classes II, abstract elementary classes. In J.T. Baldwin, editor, Classification theory (Chicago, IL, 1985), pages 419-497. Springer, Berlin, 1987. paper 88: Proceedings of the USA-Israel Conference on Classification Theory, Chicago, December 1985; volume 1292 of Lecture Notes in Mathematics. 
[22] R.L. Vaught. Denumerable models of countable theories. In Infinitistic Methods, Proc. Symp. Foundations of Math., Warsaw, 1959, pages 303-321. Państwowe Wydawnictwo Naukowe, Warsaw, 1961.

[23] B.I. Zilber. A categoricity theorem for quasiminimal excellent classes. In Logic and its Applications, Contemporary Mathematics, pages 297-306. AMS, 2005. 